\title{
Evaluation of prevalence of different types of cancer and its chemotherapy in various ethnic groups of Pakistan: A retrospective study
}

\author{
Danish Ali', Syed Baqir Shyum Naqvi², Muhammad Iqbal Nasiri ${ }^{\circledR 3 *}$, \\ Kamran Ahmed ${ }^{1}$, Kamran Zaheer², Muhammad Azeem³, \\ Muhammad Masoom Akhtar ${ }^{3}$ \\ ${ }^{1}$ Department of Pharmaceutics, Faculty of Pharmacy and Pharmaceutical Sciences, \\ University of Karachi, Pakistan, ${ }^{2}$ Departments of Pharmaceutics, Faculty of Pharmacy, \\ Hamdard University, Karachi, Pakistan, ${ }^{3}$ Departments of Pharmaceutics, Hamdard Institute \\ of Pharmaceutical Sciences, Hamdard University Islamabad Campus, Pakistan
}

\begin{abstract}
The objective of the study was to evaluate different types of cancer and its chemotherapy in various ethnic groups of Pakistan. Ethnic groups includes, Pukhtoons, Punjabis, Sindhis, Muhajirs, Siraikis, Memoons, Hazaras, Hindkos, Baltis, Gilgitis, Kashmiris, and Afghanis. The data was collected from well reputed hospitals located in the different provinces of Pakistan. The collected data was taken from 15 hospitals where around 8500 patients visited during 2010 to 2017. From the visited patients, 8356 were analyzed for their ethnicity, age and sex while, 144 patients (male 77 and female 67) were excluded from analysis due to incomplete information or loss of follow-up. Among 8356 patients, 3762 were male (45\%) whereas, 4594 were female (55\%). The chemotherapy was carried out as per National Comprehensive Cancer Network guidelines (NCCN- guidelines). The most common five prevalent cancer among these ethnic groups were Head and Neck, Blood, Respiratory, Genito-urinary and Breast cancer. The most common cancer in female was breast cancer while, head and neck cancer was more prevalent in male. It can be concluded that the prevalence of cancer in Pakistan is very alarming, which may be due to lack of awareness, illiteracy, lack of national cancer control programs, and economics issues.
\end{abstract}

Keywords: Cancer. Chemotherapy. Ethnic groups. Pakistan.

\section{INTRODUCTION}

Humans have been aware of cancer since ancient time in spite of the fact that the word of cancer was not used. The term cancer was first used by the Greek physician Hippocrates (460-370 BC). The terms carcinos and carcinoma was used to depict non-ulcer shaping and ulcer shaping tumors individually. Galen was the primary researcher who utilized word "oncos" (In Greek, swelling) for description of tumors whereas, the term of Hippocrate and Celcus are utilized to

*Correspondence: M. I. Nasiri. Departments of Pharmaceutics. Hamdard Institute of Pharmaceutical Sciences. Hamdard University Islamabad Campus, Pakistan. Tel.: +923343377642. Email: iqbalnasiri@hotmail.com describe malignant tumors till presently (Jemal et al., 2008). There are many types of cancers which effect the human body and are named usually by the body part that they originated. $90-95 \%$ causes of cancer is genetic mutations from environmental factors (tobacco, diet, obesity, infection, radiations, stress etc.) which are not inherited genetically and the rest $5-10 \%$ are due to inherited genetics (Anand et al., 2008)2008. In Western Europe $9.99 \%$ of cancers in male and $3 \%$ in female is due to alcohol exposure, particularly liver and gastric track (Schütze et al., 2011). Most cancers are analyzed by the appearance of signs/indications, through screening, examination of tissue, or x-rays, CT scans and endoscopy. Globally as well as in Pakistan, cancer is one of the major cause of death. It is the second most 
common cause of death in worldwide (Cokkinides et al., 2005) and its prevalence may extend from 6.1 to 10.7 million in 2030 (Sankaranarayanan et al., 2014). The prevalence rate is anticipated with the rate of 150,000 cancers each year (Noronha et al., 2012). Cancer may be treated by surgery, chemotherapy, radiation treatment, hormonal treatment, focused on treatment (counting resistant treatment) and manufactured lethality. The choice of treatment depends upon the condition, stage and portion of body. The ultimate goal is to remove the cancer completely without damaging the other parts of body. As the cancer is a group of diseases, so the single therapy is not possible. However, there is a clear message of hope as it is largely preventable. If we have adequate data about cancer patients, then by applying appropriate measures, we can reduce the malignancy burden in Pakistan as well as globally (Yeole, 2006). Cancer is basically a class of disorder marked by uncontrolled proliferation of cells which have the potential to interfere with different systems of body. Tumors that reside only in a specified location and shows restricted growth, are commonly characterized as benign tumors. When tumor cells grow and effectively spread to other body parts and potentially invade and damage healthy tissues they show various degrees of malignancy. Cancer may be caused by different factors like gene mutations, carcinogens and some medical factors. Cancer management and chemotherapy protocols depend on the progression and site where it develops. The most commonly diagnosed cancers in Pakistan are breast, lung, liver, colorectal, prostate, head and neck carcinoma. In the current study, an efforts has been made to evaluate the prevalence of cancer and its chemotherapy in different ethnic groups of Pakistan, in order to take preventive measures.

\section{MATERIAL AND METHODS}

\section{Protocol approval}

The study protocol was approved by the Board of Advanced Studies and Research (BASR), University of Karachi, Karachi.

\section{Design of study}

A retrospective study was conducted for the chemotherapeutic evaluation of cancer to determine actual incidences in male and female genders and their ethnic relation in Pakistan. For this purpose, an extensive survey was conducted to different hospitals of province of Punjab, Sindh and KPK, Pakistan registering cases of cancer.

During the study, the incidences of cancer, types, diagnosis year, age, gender, marital status, ethnic group, occupations and other risk factors were investigated. As per guidelines given by international classification coding system of WHO, different types of cancers were classified (ICD-10).

\section{Data collection}

Strategy has been designed and implemented to collect the data from the oncology departments of the 15 targeted government hospitals, semi government hospitals and private hospitals across the Pakistan. Data were collected after taking permission officially within allocated period i.e. from January 2010 to December 2017.

\section{Sample size \& selection}

Size of the sample was based on the data available in Hospitals. Approximately, 8500 patients with histopathological findings of cancer were selected for analysis, among which 8356 patients were included in final evaluation of incidences while, 144 patients were excluded due to reasons mentioned in exclusion criteria.

\section{Inclusion criteria for analysis}

- Patients diagnosed with cancer histologically and cytologically were included.

- Type of cancer clearly mentioned.

- Proven malignancy by tests.

- Availability of ethnic group \& geographical belongings of the patients.

- The age \& gender of patient documented.

\section{Exclusion criteria for analysis}

- Patient with preliminary diagnosed for cancer.

- Patient's objective findings was not done/ not histologically and cytologically proved.

- Type of cancer NOT clearly mentioned/confirmed.

- Accessibility of incomplete data of patients like, ethnic community, age, sexual orientation \& belonging to geographic area.

- Children $\leq 12$ years. 


\section{Epidemiological and statistical analysis}

On the basis of data obtained from patient's records, stratification was done to determine the incidences of cancer in each gender and ethnic groups like, Pukhtoons, Punjabis, Sindhis, Muhajirs (Urban sindhi), Siraikis, Memoons, Hazaras, Hindkos, Baltis, Gilgitis, Kashmiris, Afghanis, and unknown ethnicity of province of Punjab, Sindh and KPK. The data was statistically evaluated by using SPSS software. The analysis was done for the fractions or proportions of cancer, mean age, standard deviation and co-efficient of variation ( $\%$ RSD). The distribution curve was plotted to obtain high risk age and ranking of cancers in total population as well as gender wise in each ethnic background, estimated by SPSS version 21.

\section{RESULTS AND DISCUSSION}

\section{Demographic data of patients}

The present study indicated the chemotherapy and incidence of cancer among all ethnic groups in Pakistan. 8500 patients visited fifteen hospitals all across the Pakistan, out of which 8356 patient's data were considered. Among 8356 patients, 4594 (55\%) were female and 3762 (45\%) were male. Approximately, 144 (1.69\%) patients were excluded due to incomplete information. The overall frequency of distribution of patients belong to various ethnic groups were observed as Afghani 4.88\%, Balochi $3.89 \%$, Balti $2.49 \%$, Gilgiti $2.46 \%$, Hazara 3.48\%, Hindko 3.39\%, Memon 2.26\%, Punjabi $24.47 \%$, Pushto 16.69\%, Saraiki 9.23\%, Sindhi $11.65 \%$ and Urban sindhi (Muhajir) $15.09 \%$. The demographic study of different ethnic groups are given in Table 1.

Table 1: Demographic study of different ethnic groups

\begin{tabular}{llcc}
\hline \multirow{2}{*}{ Ethnic Group } & \multicolumn{1}{c}{ Gender } & No. cases & \% distribution \\
& & & \\
\hline Afghani & Male & 186 & 2.22 \\
& Female & 222 & 2.66 \\
Balochi & Male & 172 & 2.06 \\
& Female & 153 & 1.83 \\
Balti & Male & 115 & 1.38 \\
& Female & 93 & 1.11 \\
Gilgiti & Male & 98 & 1.17 \\
& Female & 108 & 1.29 \\
Hazara & Male & 119 & 1.42 \\
& Female & 172 & 2.06 \\
Hindko & Male & 138 & 1.65 \\
& Female & 145 & 1.74 \\
Memon & Male & 97 & 1.16 \\
& Female & 92 & 1.1 \\
Punjabi & Male & 920 & 11.01 \\
& Female & 1125 & 13.46 \\
Pushto & Male & 637 & 7.62 \\
& Female & 758 & 9.07 \\
Saraiki & Male & 360 & 4.31 \\
& Female & 411 & 4.92 \\
Sindhi & Male & 464 & 5.55 \\
Urban sindhi & Female & 510 & 6.1 \\
(Muhajir) & Male & 456 & 5.46 \\
\hline & Female & 805 & 9.63 \\
\hline & & $\mathbf{8 3 5 6}$ & $\mathbf{1 0 0}$ \\
\hline
\end{tabular}




\section{Prevalence of cancers}

Figure 1 shows the types of cancer, number of cases and \%age distribution reported from year 2010 to 2017. According to the data, Head and Neck cancer was the most prevalent type (19.9\%), followed by breast cancer (19.6\%). The incidence of blood cancer was observed about $14.9 \%$ while, Integumentary cancer was the least one $(0.5 \%)$.

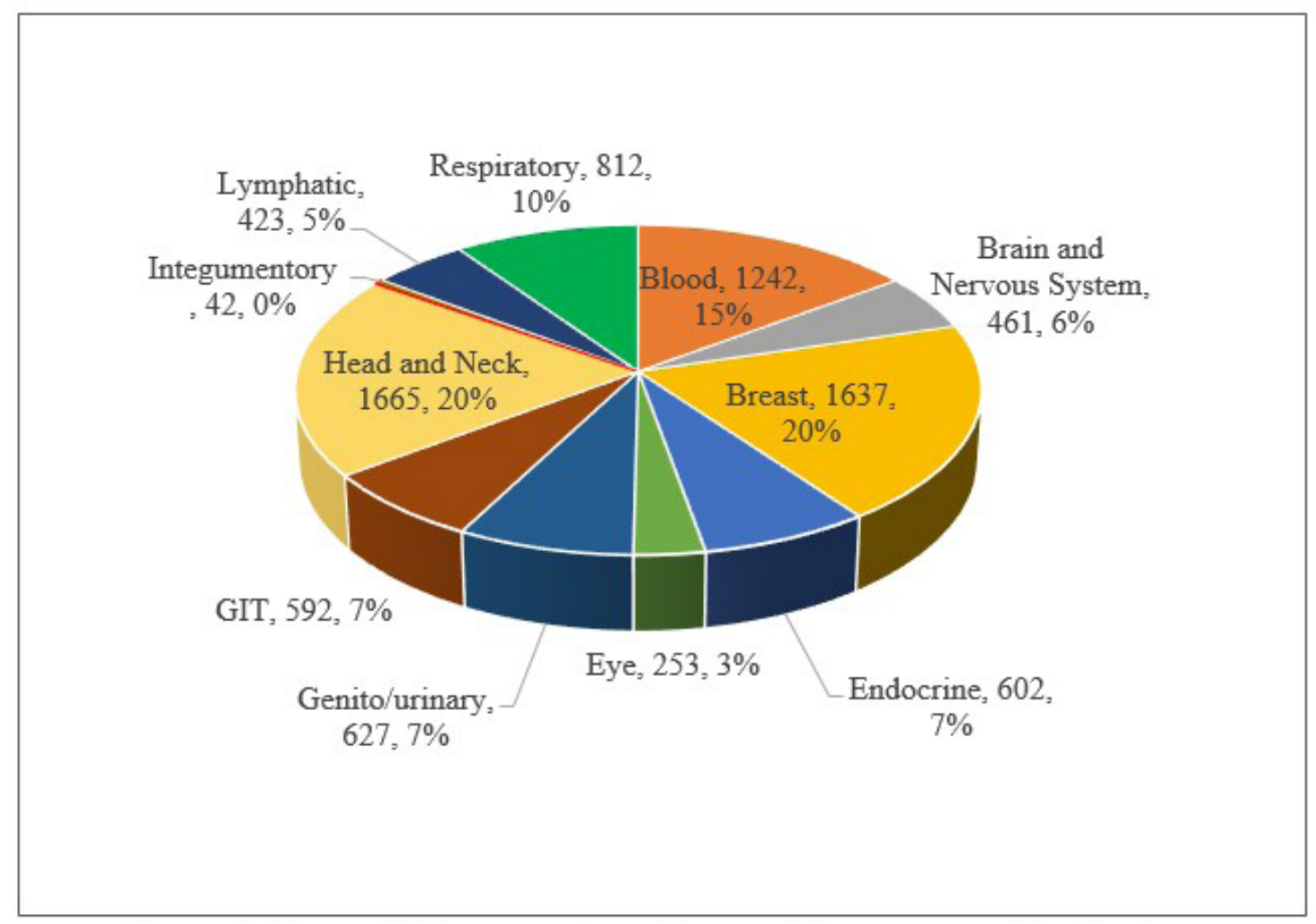

Figure 1 - Types of cancer and no of cases with percentage distribution.

Figure 2 indicates the prevalence/frequency of cancer in different age groups of both genders. Patients having age from 41-50 years showed maximum risk of cancer in these ethnic groups (2209 cases), whereas, age ranges from 91 - 100 years, showed least prevalent (3, case only).

The most prevalent population/ ethnic group was Punjabi $(24.47 \%)$ while, the least prevalent population was Memons (2.26\%). Thus, the screening of cancers should be done in this groups to reduce the burden of cancer in Pakistan and its chemotherapy. In addition to this, the other ethnic groups require more resources for screening because of variability of high risk age included, Sindhi, Muhajirs (Urban sindh) and Puhktoon males (high risk age $51-60$ years), so these age groups should be targeted for the screening and monitoring of cancers other than $41-50$ years age group.
The study showed that highest incidences of head and neck cancer (especially oral cavity squamous cell carcinoma) was recorded among male, while, breast cancer among female genders. The risk factors may be due to excessive use of tobacco or cigarette, gutka, pan, betel nut, menpori, suparis, naswar and acid reflux disease. It has been reported that human papilloma virus type 16 and Epstein barr virus are also associated with the development of oral squamous cell carcinoma (D’Souza et al., 2011; Khaliq et al., 2013). Another study indicated that 36\% male and $44 \%$ female in Karachi (highest populated city of Pakistan) chew either pan alone or pan with tobacco and almost half of the cancers in male and quarter of cancers in female are because of tobacco. The screening should be done for all male and female at high risk having age $41-50$ years. Head and Neck (HN) cancer is the 
foremost regularly distinguished cancer over the globe and is considered as $8-10 \%$ of all cancers in Southeast Asia. Typically, primarily credited to tobacco, areca nut, liquor, etc. Oral cancers are most common among all head and neck squamous cell cancers (Joshi et al., 2014).

Table II shows gender wise prevalence of different types of cancer in various provinces of Pakistan.

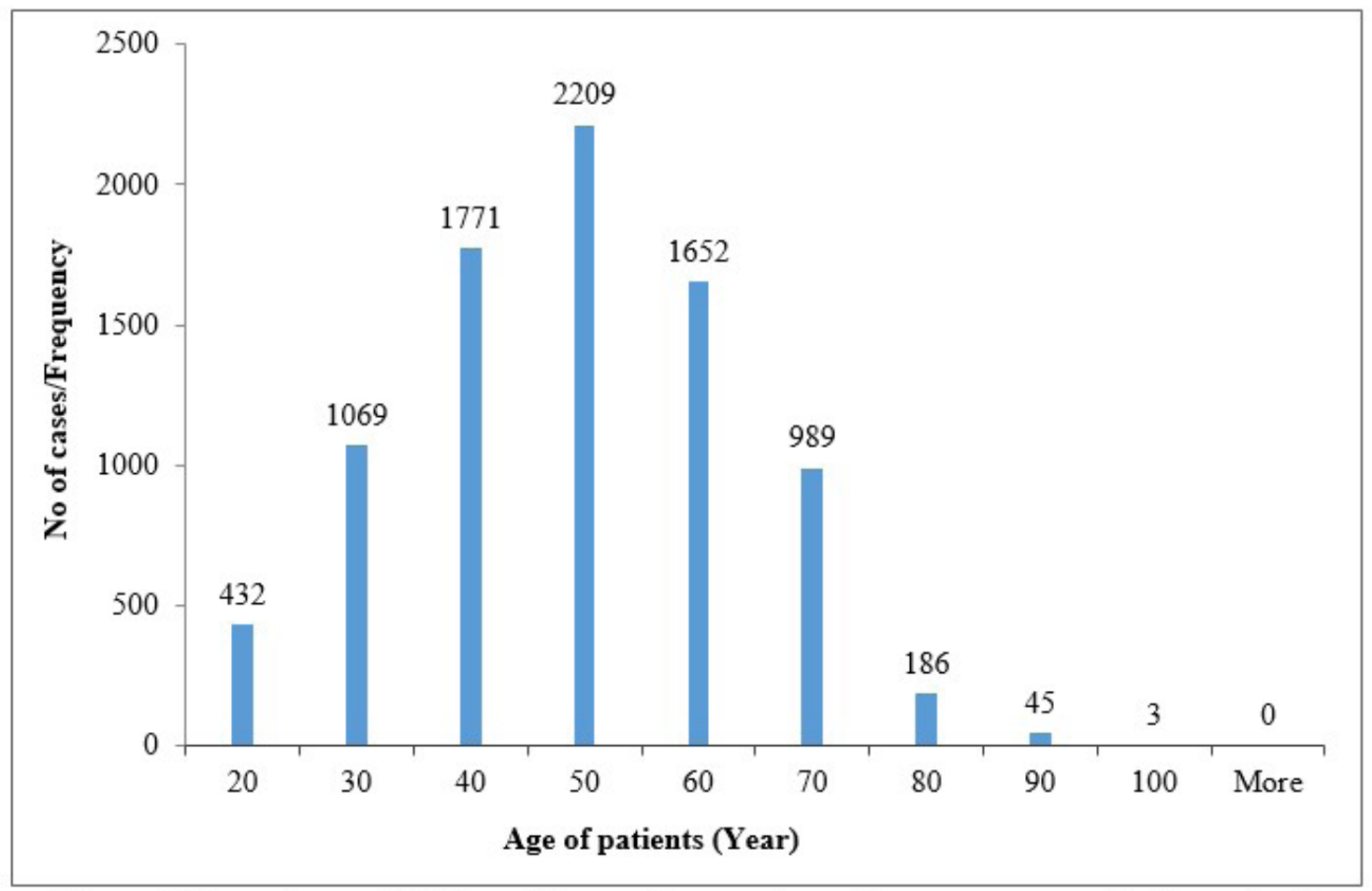

Figure 2 - Prevalence of different types of cancer in various age groups (Both genders).

In Sindh Province, the prevalence of breast cancer was noted most common among females of different ethnic group such as, Memoons (48.9\%), Muhajirs females (35.16\%) and Sindhis female (27.3\%). In Punjab Province, these incidences were observed as, Punjabis (39.1\%), saraikis female (33.6\%). Whereas, among KPK Province, the prevalence of breast cancer in female were noted as Hazaras (40.7\%), Hindko (35.2\%) and Pukhtoon (37.5\%). The most affected female age groups were $41-50$ and 51- 60 years. In Gilgit Baltistan (GB) province (Baltis), the incidence in female was reported very low i.e. (23.7\%), while in Gilgitis, it was reported as $28.70 \%$. The main causes of these cancers reported by some authors are socio-economic conditions, illiteracy and lack of medical facilities (Ayub et al., 2011; Chang et al., 2011; Mousa et al., 2011; Schlichting et al., 2012). At some stage of life, 1 in 9 Pakistani women has become the patient of breast cancer (Sohail et al., 2007). In Sindh province, the incidence of respiratory cancer in different male ethnic groups were recorded as, memoons $(28.7 \%)$, sindhis $(16.4 \%)$ and urban sindhis (Muhajirs) (9.6\%), whereas, in Punjab province, this incidence was observed as punjabis (16.4\%) and saraikis male $(15.3 \%)$. In KPK Province, the prevalence in Hazaras was $15.1 \%$, in Hindko $14.9 \%$ and in pukhtoon $13.9 \%$. Low incidences were reported in GB Province, like in male ethnic group of gilgiti was $4.2 \%$. Martins et al. reported that lung cancer is the third most leading cause of cancer death in Pakistan as 4.6\% new cases and $5.9 \%$ deaths occurring in 2012 (Martins et al., 2016).

The incidence of blood cancer among Sindhi male (33\%), Sindhi female (22.9\%), urban sindhis (Muhajirs) male $(17.3 \%)$, urban sindh female $(9.8 \%)$, Baloch male (13.5\%), Baloch female (13.7\%) Pukhtoon male (17.8\%), 
Pukhtoon female (12.5), Punjabi male (17.6\%), Punjabi female (8.9\%), Baltis male (19.1\%) and Baltis female (15.1\%), were recorded. This data indicated that blood cancer was more prevalent in male gender than female. Non Hodgkins Lymphoma (NHL) was the sixth most common cancers in female of Gilgit and Baltistan. Similar findings were also reported by Zeba et al. 1999. The prevalence of genito-urinary malignancy were observed higher in Sindh Province of different ethnic groups like, urban sindhi males (10.1\%) and sindhis male (7.3\%). In Punjab Province, it was 6.6\% in Punjabis male and $8.3 \%$ in saraikis male, whereas, in KPK Province, the incidence was $6.3 \%$ in pukhtoon males and 3.4\% in Hazara. While, in Memoon male it was $3.4 \%$. The data of the present study was supported by Shi et al. 2012, who reported cervical cancer (Shi et al., 2012). The prevalence of gastrointestinal cancers were reported, in afghani refugees $2.3 \%$, in phaktoons $9.1 \%$, in Gilgitis $8.8 \%$, whereas, in urban sindh (Muhajirs) it was $8.2 \%$. However, among all types of gastrointestinal cancers, the incidence of colorectal cancer was highest. The incidences were observed high in Punjabi males (10.78\%), Pukhtoon male (7.49\%), Sindhi male (5.46\%) and in urban sindhi males $(5.36 \%)$, which represents that population living in Sindh province have high incidences.

Table 2: Prevalance of types of cancer ( $\%$ distribution) in various provinces of Pakistan

\begin{tabular}{llcccc}
\hline Types of Cancer & Gender & $\begin{array}{c}\text { Punjab } \\
(\mathbf{\%})\end{array}$ & $\begin{array}{c}\text { Sindh } \\
\mathbf{( \% )}\end{array}$ & $\begin{array}{c}\text { KPK } \\
\mathbf{( \% )}\end{array}$ & $\begin{array}{c}\text { Gilgit-Baltistan } \\
\text { (\%) }\end{array}$ \\
\hline Blood & Male & 17.15 & 20.81 & 20.45 & 17.37 \\
\multirow{3}{*}{ Brain and Nervous System } & Female & 8.91 & 15.17 & 14.62 & 11.73 \\
& Male & 13.50 & 0.43 & 2.23 & 19.25 \\
Breast & Female & 7.34 & 0.00 & 0.00 & 15.31 \\
& Male & 0.00 & 0.00 & 0.00 & 6.57 \\
Eye & Female & 32.62 & 39.59 & 39.65 & 27.04 \\
& Male & 5.28 & 0.17 & 0.00 & 5.63 \\
Genitoiurinary & Female & 5.42 & 0.79 & 0.47 & 6.12 \\
GIT & Male & 7.40 & 8.77 & 5.24 & 7.98 \\
& Female & 6.22 & 7.94 & 11.35 & 5.10 \\
Head and Neck & Male & 2.94 & 14.53 & 15.99 & 2.82 \\
& Female & 3.34 & 7.05 & 9.24 & 3.06 \\
Lymphatic & Male & 27.82 & 26.06 & 25.95 & 20.66 \\
& Female & 15.52 & 13.05 & 11.81 & 18.37 \\
Integumentary & Male & 5.01 & 7.48 & 5.24 & 6.57 \\
& Female & 6.45 & 2.29 & 1.17 & 6.63 \\
Endocrine & Male & 0.05 & 0.86 & 2.49 & 0.00 \\
& Female & 0.08 & 0.18 & 0.94 & 0.00 \\
Respiratory & Male & 5.99 & 7.22 & 7.99 & 4.69 \\
& Female & 5.11 & 12.43 & 8.54 & 4.08 \\
& Male & 14.86 & 13.67 & 14.42 & 8.45 \\
& Female & 8.99 & 1.50 & 2.22 & 2.55 \\
\hline & & & & &
\end{tabular}




\section{Chemotherapy regimens}

The chemotherapy carried out in case of breast cancer across the Pakistan was 6 cycles of FAC therapy (5-fluorouracil, Adriamycin/doxorubicin, cyclophosphamide) and showed good response as they followed the international NCCN guideline. Tariq et al., confirmed the data (Tariq et al., 2015). Whereas, in case of head and neck cancers, chemotherapy regimens followed as cisplatin $100 \mathrm{mg} / \mathrm{m}(2)$ in $3-\mathrm{h}$ infusion, day 1 and 5-FU $1000 \mathrm{mg} / \mathrm{m}(2)$ in 24-h infusion, days 1-5. Treatment was repeated every 21 days. A total of 154 treatment courses (3.5 per patient, ranged 1-10) were administered. Myelotoxicity, nausea and vomiting were the major treatment complications. Chemotherapy with cisplatin and 5-FU combination is effective in patients with advanced squamous cell oral cancer and appears to improve the survival of patients who have a good response (Andreadis et al., 2003). The chemotherapy in respiratory cancer was carried intraperitoneally or systemically, includes, Cisplatin, Mitomycin C, Fluorouracil, Doxorubicin, and/or Paclitaxel. Cisplatin is the most studied drug, with activity in $25 \%$ of patients (Bano et al., 2013). The chemotherapeutic regimens followed for blood cancer included $\mathrm{CHOP}$ (Cyclophosphamide, Adriamycin, Prednisolone, Vincristine), BACOP (Bleomycin, Adriamycin, Cyclophosphamide, Prednisolone, Vincristine), CVP (Cyclophosphamide, Vincristine, Prednisolone), ProMACE (Procarbazine, Methotrexate, Adriamycin Cyclophosphamide, Etoposide) and Cytarabine. Aziz et al., and NCCN guideline also supported the data (Aziz et al., 1999). The chemotherapy carried out to treat Genito-Urinary cancer were fuorouracil, oxalipatin, irinotecan, cetuximab, 5-FU. These agents may be used alone or in combination form such as FOLFIRI, FOLFOX, XELOX, irinotecan/ oxalipatin and uraciltegafur plus leucovorin (UFT/LV) and among these regimens, FOLFOX is the most frequent and effective regimen (Tariq et al., 2015).

Chemotherapeutic regimens used in gastrointestinal cancer included, double contrast barium enema chemotherapy and radiotherapy i.e. (1) fluorouracil (5-FU) alone or in combination with radiotherapy, (2) 5-fluorouracil and folinic acid, (3) irinotecan (4) oral capecitabine, (5) irinotecan, (6) combination of irinotecan, 5-fluorouracil and 5-folinic acid (Tariq et al., 2015).

\section{CONCLUSIONS}

The objective of the current study was to evaluate various kinds of cancer and its chemotherapy in different ethnic groups of Pakistan, such as, Pukhtoons, Punjabis, Sindhis, Urban sindhis (Muhajirs), Siraikis, Memoons, Hazaras, Hindkos, Baltis, Gilgitis, Kashmiris, and Afghanis. It was concluded that breast cancer was the most common cancer in female while, head and neck cancer was more prevalent in male among all ethnic groups. The second most prevalent types of cancer in both genders were, blood and respiratory cancer. The frequency or no of cases of cancers were more common in $40-60$ years of age group and less common above 85 years. The chemotherapy regimens followed by all hospitals complied the NCCN guideline. Pakistan, is an under developed country, where the burden of the disease on the health care system is enormous. Therefore, different strategies has to be developed to overcome the burden of cancer in terms of financial and other losses. It has become challenging to form a National Cancer Control Program (NCCP) for better checking and disease control. The government, $\mathrm{WHO}$ and private sector NGOs should provide necessary resources to develop strategies for screening, prevention, diagnosis and treatment of cancer especially breast cancer, head and neck cancer and other prevailing cancers. Few cancers can be reduced by proper screening such as mammography can helps to screen out the breast cancer and Pap test/screening can prevent cancer of cervix at earlier stage. Moreover, immunization/ vaccine is also available which can prevent both cervix cancer and liver cancer by proper vaccination. Most of the population cannot manage this screening program since it is very costly. The only procedure is breast self-examination (BSE) to check one-self month to month. Prepared restorative specialists and medical attendants at wellbeing centers educate the ladies, how to utilize breast self-examination method once a month. Therefore, they can use easily this technique by themselves to detect breast cancer at early stage.

\section{ACKNOWLEDGEMENTS}

Authors are thankful to hospitals and Department of Pharmaceutics, Faculty of Pharmacy University of Karachi for providing data facilities. 


\section{CONFLICT OF INTEREST STATEMENT}

Authors declared no conflict of interests.

\section{FUNDING}

For this projects no funding was available.

\section{REFERENCES}

Anand P, Kunnumakara AB, Sundaram C, Harikumar KB, Tharakan ST, Lai OS, et al. Cancer is a preventable disease that requires major lifestyle changes. Pharm Res. 2008;25(9):2097-2116.

Andreadis C, Vahtsevanos K, Sidiras T, Thomaidis I, Antoniadis K, Mouratidou D. 5-Fluorouracil and cisplatin in the treatment of advanced oral cancer. Oral Oncol. 2003;39(4):380-385.

Ayub SG, Ayub T, Khan SN, Rasool S, Mahboob-ul-Hussain WK, Khursheed A. Epidemiological distribution and incidence of different cancers in Kashmir Valley-2002-2006. Asian Pac J Cancer Prev; 2011;12(7):1867-1872.

Aziz Z, Rehman A, Akram M, Saeed A. Non-Hodgkin's lymphoma in Pakistan: a clinicopathological profile of 175 patients. JPMA J Pak Med Assoc. 1999;49(1):11-15.

Bano N, Najam R, Mirza T, Hassan S. Review of colorectal carcinoma and specific disease features in the population of Pakistan. Asian J Pharm Clin Res. 2013;6(Suppl 1): 1-5.

Chang G, Chan CW, Hartman M. A commentary on delayed presentation of breast cancer in Singapore. Asian Pacific J Cancer Prev. 2011;12(6):1635-1639.

Cokkinides V, Albano J, Samuels A, Ward M, Thum J. American cancer society: Cancer facts and figures. Atlanta: American Cancer Society; 2005.

D'Souza G, Dempsey A. The role of HPV in head and neck cancer and review of the HPV vaccine. Prev Med. 2011;53(Suppl 1):S5-S11.

Jemal A, Siegel R, Ward E, Hao Y, Xu J, Murray T, Thun MJ. Cancer statistics 2008. CA Cancer J Clin. 2008;58(2):71-96.

Joshi P, Dutta S, Chaturvedi P, Nair S. Head and neck cancers in developing countries. Rambam Maimonides Med J. 2014;5(2):e0009.
Khaliq SA, Naqvi SB, Fatima A. Retrospective study of cancer types in different ethnic groups and genders at Karachi. Springerplus. 2013;2(1):118.

Martins T, Hamilton W. The influence of ethnicity on diagnosis of cancer. Oxford: University Press UK; 2016.

Mousa SM, Seifeldin IA, Hablas A, Elbana ES, Soliman AS. Patterns of seeking medical care among Egyptian breast cancer patients: relationship to late-stage presentation. Breast. 2011;20(6):555-561.

Noronha V, Tsomo U, Jamshed A, Hai M, Wattegama S, Baral R, Piya M, Prabhash K. A fresh look at oncology facts on south central Asia and SAARC countries. South Asian J Cancer. 2012;1(1):1-4.

Sankaranarayanan R, Ramadas K, Qiao Y-1. Managing the changing burden of cancer in Asia. BMC Med. 2014;12(1):3.

Schlichting JA, Soliman AS, Schairer C, Banerjee M, Rozek LS, Schottenfeld D, et al. Association of inflammatory and noninflammatory breast cancer with socioeconomic characteristics in the Surveillance, Epidemiology, and End Results Database, 2000-2007. Cancer Epidemiol Biomarkers Prev. 2012;21(1):155-165.

Schütze M, Boeing H, Pischon T, Rehm J, Kehoe T, Gmel $\mathrm{G}$, Olsen A, et al. Alcohol attributable burden of incidence of cancer in eight European countries based on results from prospective cohort study. BMJ. 2011;342:d1584.

Shi JF, Canfell K, Lew JB, Qiao YL. The burden of cervical cancer in China: synthesis of the evidence. Int $\mathrm{J}$ Cancer. 2012;130(3):641-652.

Sohail S, Alam SN. Breast cancer in pakistan - awareness and early detection. J Coll Physicians Surg Pak. 2007;17(12): 711-712.

Tariq A, Majeed I, Khurshid A. Types of cancers prevailing in Pakistan and their management evaluation. Asian Pacific J Cancer Prev. 2015;16(9):3605-3616.

Yeole BB. Role of the cancer registries in determining cancer mortality in Asia? Asian Pacific J Cancer Prev APJCP. 2006;7(3):489-491.

Received for publication on $29^{\text {th }}$ October 2018 Accepted for publication on $14^{\text {th }}$ January 2019 